\title{
Nanoscale redox reaction unlocking the next- generation low temperature fuel cell
}

\author{
Qi Fan ${ }^{1}, *$ Shicheng Yan ${ }^{2}, *$, Hao Wang ${ }^{3}$ \\ ${ }^{1}$ College of Material Science and Technology, Southeast University, Nanjing 210096, Jiangsu, China. \\ ${ }^{2}$ Jiangsu Key Laboratory for Nano Technology, Eco-materials and Renewable Energy Research Center (ERERC), Collaborative \\ Innovation Center of Advanced Microstructures, College of Engineering and Applied Sciences, Nanjing University, Nanjing \\ 210093, Jiangsu, China. \\ ${ }^{3}$ Jiangsu Provincial Key Laboratory of Solar Energy Science and Technology/Energy Storage, Research Center, School of Energy \\ and Environment, Southeast University, Nanjing 210096, Jiangsu, China.
}

^Correspondence to: Prof. Qi Fan, College of Material Science and Technology, Southeast University, No. 2 Si Pai Lou, Nanjing 210096, Jiangsu, China. E-mail: fanqi1984@126.com; Prof. Shicheng Yan, Jiangsu Key Laboratory for Nano Technology, Ecomaterials and Renewable Energy Research Center (ERERC), Collaborative Innovation Center of Advanced Microstructures, College of Engineering and Applied Sciences, Nanjing University, No. 22 Hankou Road, Nanjing 210093, Jiangsu, China. E-mail: yscfei@nju.edu.cn

How to cite this article: Fan Q, Yan S, Wang H. Nanoscale redox reaction unlocking the next-generation low temperature fuel cell. Energy Mater 2022;2:200002. https://dx.doi.org/10.20517/energymater.2021.26

Received: 8 Dec 2021 First Decision: 8 Dec 2021 Revised: 16 Dec 2021 Accepted: 21 Jan 2022 Published: 7 Feb 2022

Academic Editor: Yuping Wu Copy Editor: Xi-Jun Chen Production Editor: Xi-Jun Chen

Solid oxide fuel cells (SOFCs) represent a next-generation energy platform technology. Lowering the operating temperature has become a hot topic in SOFC research and is pivotal to their commercialization, since lower temperatures improve the sealing and durability, as well as reducing costs. However, the lower oxide-ion diffusion and transport in the electrolyte and electrodes at low temperatures seriously inhibit the electrochemical performance and practical applications of SOFCs. Therefore, the design of new structural and functional materials with high ionic conductivity and high electrocatalytic activity is crucial to the development of next-generation low temperature fuel cells.

To face this challenge, Zhu et al. ${ }^{[1]}$ have developed semiconductor-ionic materials (SIMs), which enable the production of new advanced SOFCs known as semiconductor membrane fuel cells (SMFCs), which link semiconductor physics and fuel cell electrochemistry at the nanoscale. In contrast to traditional SOFCs, the SMFC concept is proposed to replace the traditional electrolyte by a SIM or semiconductor membrane and it can deliver superior performance even at a lower temperature range $\left(300-500{ }^{\circ} \mathrm{C}\right)$. 


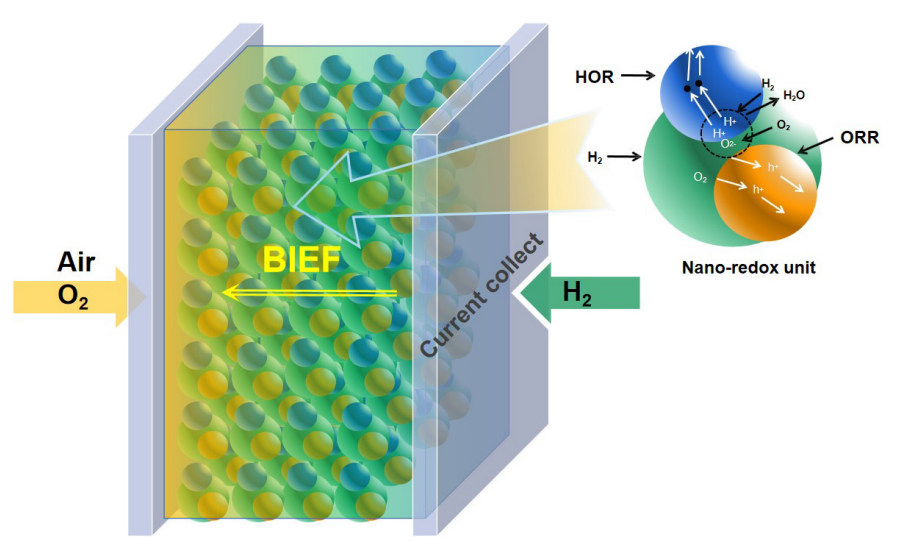

Figure 1. Nano-redox units used to build a macroscale fuel cell device. HOR: Hydrogen oxidation reaction; ORR: oxygen reduction reaction.

In such a special design, the p-and n-type layers/zones of the SMFC can be regarded as the cathode and anode of a traditional SOFC, respectively, where the electrolyte layer used to separate the hydrogen oxidation reaction (HOR) and oxygen reduction reaction (ORR) is not required. Meanwhile, the p-n junctions of SMFCs can realize the charge separation function under a built-in or internal electric field (IEF). They can block the electron flow to prevent the short-circuiting risk of SMFCs. This indicates that a physically separated electrolyte layer may not actually be an indispensable component for SOFC operation. In fact, the feasibility of the single-layer fuel cell constructed by a bulk p-n heterojunction consisting of ionic and $\mathrm{p}$ - and n-type semiconductor nanocomposite materials and a double layer fuel cell by the cathode and anode with a planar $\mathrm{p}-\mathrm{n}$ junction has been demonstrated earlier ${ }^{[1-5]}$.

Furthermore, this strategy can integrate the cathode, electrolyte and anode into a nano-redox unit consisting of ionic and $n$ - and p-type particles. This means that the nano-redox unit ${ }^{[6]}$, which suggests that SMFC devices can be constructed at the nanoparticle scale, as shown in Figure 1. By doing so, it can obviously promote the fuel cell HOR and ORR reactions in situ without the traditional separation by the electrolyte layer. In addition, the IEF caused by the $\mathrm{p}-\mathrm{n}$ junction can drive the transport of the ions, e.g., $\mathrm{H}^{+}$ and $\mathrm{O}^{2-}$, thus increasing the ionic conductivity and output current/power. All of these can minimize or even avoid the interfacial polarization that is critical for traditional SOFCs, especially those operating at low temperature. Moreover, the electrolyte limitations of SOFC technology can be overcome to bring about a new pathway for SOFC commercialization.

Finally, this perspective focuses on the nano-redox mechanism and generation principle. Zhu et al. ${ }^{[7]}$ have presented a next-generation fuel cell technology using a triple conducting oxide (TCO) single-layer device, which is able to conduct three mobile charge carriers, thereby exhibiting three types of conductivities: oxygen ions, electrons and protons. TCOs are very useful for various scientific areas and potential applications in electrochemical devices, such as membranes and reactors for hydrogen or oxygen separation, or electrodes for SOFCs and protonic ceramic fuel cells. The proposed TCO single device will not only benefit fuel cells but is also meaningful for other relevant energy technologies, such as water electrolysis, $\mathrm{CO}_{2}$ electrochemical reduction and electrochemical nitrogen fixation.

\section{DECLARATIONS}

\section{Authors' contributions}

Wrote the manuscript: Fan Q, Yan S 
Discussed and commented on the manuscript: Fan Q, Yan S, Wang H

\section{Availability of data and materials}

Not applicable.

\section{Financial support and sponsorship}

Fan Q is thankful for the National Science Foundation of China (Grant number 51772080). This work was also supported by the National Natural Science Foundation of China (Grant number 51877045), the Fundation from State Key Laboratory of Materials Oriented Chemical Engineering (KL19-09) the Fundamental Research Funds for the Central Universities.

\section{Conflicts of interest}

All authors declared that there are no conflicts of interest.

\section{Ethical approval and consent to participate}

Not applicable.

\section{Consent for publication}

Not applicable.

\section{Copyright}

(c) The Author(s) 2022.

\section{REFERENCES}

1. Zhu B, Raza R, Abbas G, Singh M. An electrolyte-free fuel cell constructed from one homogenous layer with mixed conductivity. $A d v$ Funct Mater 2011;21:2465-9. DOI

2. Whitchurch A, Armstrong A, Newton A. Our choice from the recent literature. Nature Geosci 2011;4:497. DOI

3. Zhu B, Raza R, Qin H, Liu Q, Fan L. Fuel cells based on electrolyte and non-electrolyte separators. Energy Environ Sci $2011 ; 4: 2986$. DOI

4. Zhu B, Ma Y, Wang X, Raza R, Qin H, Fan L. A fuel cell with a single component functioning simultaneously as the electrodes and electrolyte. Electrochem commun 2011;13:225-7. DOI

5. Zhu B, Qin H, Raza R, et al. A single-component fuel cell reactor. Int J Hydrogen Energy 2011;36:8536-41. DOI

6. Zhu B, Raza R, Qin H, Fan L. Single-component and three-component fuel cells. J Power Sources 2011;196:6362-5. DOI

7. Zhu B, Lund P, Raza R, et al. A new energy conversion technology based on nano-redox and nano-device processes. Nano Energy 2013;2:1179-85. DOI 